\title{
WHEN KNOWING A GRAMMAR RULE MAKES L2 USERS NON-
} NATIVE-LIKE

\author{
Clarice Fernandes dos Santos ${ }^{1 *}$ \\ Ricardo Augusto de Souza ${ }^{1^{* *}}$ \\ Larissa Santos Ciríaco ${ }^{1 * * *}$ \\ ${ }^{1}$ Universidade Federal de Minas Gerais, Belo Horizonte, MG, Brasil
}

\begin{abstract}
In this work, we investigate the effects of transfer of training in late Brazilian Portuguese-English bilinguals, compared to natives, in relation to their processing of the English causative-have construction. Two experiments were conducted: one focused on the comprehension of the pattern, and the other focused on its production. The results of both experiments point to the fact that the grammatical rule learned by bilinguals is only 'transferred' when there is time to implement it. In the first experiment, we found no effect of training, on the contrary, bilinguals might have been affected by the SVO distribution of the corresponding construction in their L1. In the second experiment, however, we found that bilinguals had high rates of usage of the canonical causative-have form, SAuxOV. This behavior indicates that bilinguals do not implement the rule automatically. Rather, its implementation is seen in more controlled tasks. Thus, explicitly given grammatical rules seem not to be implicitly learned, as they are not automatized to be easily retrieved in real life usage. Moreover, concerning the causative-have rule, bilinguals' linguistic behavior was more similar to that of natives' when they did not implement the rule and used the SVO form with a causative sense.

Key-words: Training, Grammar Rule, Bilingualism, Causative-have Construction, Processing.
\end{abstract}

\footnotetext{
* Doutoranda em Linguística Teórica e Descritiva pelo Programa de Pós-Graduação em Estudos Linguísticos da UFMG. Seu e-mail é: clariceufmg@gmail.com. ORCID: https://orcid.org/0000-0001-6097-0058.

${ }^{* *}$ Professor Associado de Língua Inglesa. Docente do Programa de Pós-Graduação em Estudos Linguísticos da UFMG (Área de Concentração em Linguística Teórica e Descritiva) e Bolsista de Produtividade em Pesquisa pelo CNPq (Nível 2). Seu e-mail é: ricsouza.ufmg@gmail.com. ORCID: orcid.org/0000-0001-6690-3948.

${ }^{* * *}$ Professora Adjunta de Linguística da Faculdade de Letras. Docente do Programa de Pós-Graduação em Estudos Linguísticos da UFMG. Seu e-mail é: laciriaco@gmail.com. ORCID: https://orcid.org/0000-00018009-6839.
} 


\section{Introduction}

The immense availability of materials for the teaching of English grammar to L2 learners is likely to be promptly acknowledged by any English language teacher. Choices range from drill-based to genre-focused or discourse-based perspectives. Pedagogical presentations of L2 grammar may be approached with more or less accompanying metalanguage, just like the testing of grammatical accuracy is recognizable more or less explicitly in several widely accepted standardized L2 proficiency test batteries. It may be the case that the offer of instructional content covering L2 grammar is only catering for students' expectations that some sort of explicit presentation of the L2 linguistic structures should be part and parcel of language education, as much as their expectations to learn the L2 grammatical system as effectively as possible.

However, the extent to which learned pedagogical grammar rules are accessed by L2 users over the course of communicative events remains a subject of contention among second language researchers. Grammatical usage over online L2 processing events may rely mostly on implicit memory representations (Ullman, 2001), or else highly automatized routines of access to linguistic representations, which impose little demands on cognitive resources (Segalowitz, 2010). Such implicit representations and automatized access routines are not necessarily built up exclusively from the type of explicit information provided by pedagogical grammar teaching approaches. Also, it may be the case that explicit knowledge of language is only accessed in task conditions that allow sufficient time and mnemonic manipulations for the needed allocation of attentional resources (Krashen, 1994; Souza and Oliveira, 2017).

In the present study, we explore the issue of availability of L2 pedagogical grammar rules representations by examining a situation in which one such rule may actually not lead to native-like behavior. This is the case of the so-called causativehave construction of English. This construction is formed by the verb have as a light verb, followed by an NP, which is the direct object of the main verb in past participial form that follows it (SAuxOV). It may be followed by a PP headed by the preposition BY complemented by an NP, which is interpreted as the agent of the event depicted by the main verb. Therefore, in regard to its form, the causative-have construction may be viewed as a member of the family of passive constructions in English. An example of the construction is given in sentence (1):

(1) John had his bookcase made by a local carpenter.

The construction maps to a semantic reading of the clausal subject as the beneficiary of the event depicted by the main verb. Thus, the example given as sentence (1) implies that the referent of the proper noun John got the bookcase which was made by the local carpenter, instead of making the bookcase himself. As shown by Vilela (2009), several English L2 grammar textbooks suggest that this construction is obligatory for the expression of a beneficiary clausal subject 
in English. Therefore, if such pedagogical presentation is successfully learned and its representation is accessed by an L2 user of English, this user will implement a restriction to the possibility that a Subject-Verb-Object (SVO) construction may also entail such reading, as in sentence (2):

(2) I cut my hair at the usual parlor last week.

Note that the structure in sentence 2 allows for a direct nearly word-by-word translation into Portuguese: Eu cortei meu cabelo no barbeiro usual semana passada. Readers of the Portuguese sentence are likely to construe the intended meaning as the depiction of an event in which the parlor cut the clausal subject's hair, not that the referent of such subject cut his hair himself. The restrictive pedagogical rule suggesting that only the causative-have construction maps to a beneficiary subject reading can lead an L2 user who has access to such rule to avoid expression of the beneficiary subject reading by way of the sentence structure in (2). Vilela's (2009) work showed that, in a sentence completion task, this is exactly what happened for her Brazilian Portuguese-English bilingual participants. However, the bilinguals' behavior was at odds with Vilela's native English- speaking control participants, who did not show restrictions to sentences such as (2) above. Vilela interpreted her findings as a possible case of transfer of training in interlanguage configuration, that is, a detrimental effect of a flawed generalization induced by information obtained in teaching and learning circumstances (Selinker, 1972).

In the present study, we revisit Vilela's proposal and question whether the transfer of training effect will be manifest in a linguistic task that restrains the likelihood of access to a learned explicit grammar rule. Our main hypothesis, derived from the observations reported in Vilela (2009), is that if English nativespeakers do not necessarily possess the restriction implied by the pedagogical grammar rule, then Portuguese-English bilinguals are likely to have processed several instances of the construction in sentence (2) above over the course of their histories as users of English L2, in contexts where the beneficiary reading for the subject was evident. The restriction should only emerge, therefore, if the task at hand allows them to access the misleading rule of thumb.

In the following section, we discuss more details about the debate around the validity of pedagogical grammar teaching. We then pass over to a more detailed description of the causative-have construction and the meaning it entails. The subsequent section describes the experimental methods we employed to test our hypothesis, and the analyses of our observations. We conclude this paper with some considerations about the implications of the present study to L2 language education.

\section{L2 grammar acquisition: cognitive and pedagogical considerations}

The acquisition of L2 grammatical representations is a fundamental component of bilingualism. It comes as no surprise, then, that investigations in the 
discipline of second language acquisition have focused on analyses of specifically morphosyntactic accuracy of the L2 users' performance as a pivotal source of information and as the testing ground for theoretical proposals for nearly fifty years. The question of how L2 grammatical structures are acquired, or the reasons why they may fail to be acquired, lies at the heart of the conceptualization of interlanguage (Nemser, 1971; Selinker, 1972). It has continued as the cornerstone of hypotheses about the upper boundaries of L2 proficiency that have been recently put forward. One example is Slabakova's (2012) Bottleneck Hypothesis, which predicts that automatic access to inflectional morphology may be the ultimate learning barrier that many L2 learners may fail to truly trespass. Another one is Sorace's (2011) Interface Hypothesis, according to which the ultimate learning barrier, perhaps one most L2 speakers will not overcome, are structures that intermingle syntax/semantics and pragmatics.

It is therefore no coincidence that the acquisition of grammatical knowledge has also played a pivotal role in the inquiry of second language teaching pedagogy. However, as pointed out by Nassaji (2017), the main point of concern among scholars devoted to such inquiry is whether or not, under which circumstances, and to what extent, overt grammar instruction has lasting effects on the L2 learner's capacity to access grammatical knowledge for accurate performance in natural L2 communication. This has been a matter for a long-lasting debate in the field of psycholinguistically-informed second language acquisition studies, and it is perhaps one of the subjects of contention that holds the highest stakes for L2 education.

One may argue that the beginning of the debates concerning the validity of L2 grammar instruction resulted from studies of natural orders of functional morpheme acquisition that took place in the 1970s. Studies by Dulay \& Burt (1973; 1974) suggested that children learning English as a second language followed a somewhat fixed order in the acquisition of a set of morphosyntactic patterns independently of the L2 immersion and instructional contexts, and independently of the children's first language. Although the main goal of such studies was to challenge the viewpoint that L1 transfer was the sole cause of non-native-like forms produced in L2 users' speech, the authors' construal of the their findings relevance for L2 instruction is testified by the very title of their 1973 paper: "Should we teach children grammar"? The assumption that the acquisition of $\mathrm{L} 2$ inflectional morphosyntax could be guided by universal learner-internal mechanisms provided a negative answer to the title question, according to Dulay and Burt.

The findings of the natural order of morpheme acquisition made their way to Stephen Krashen's theoretical model of second language acquisition. Such model predicts that the sole mechanism driving L2 acquisition is the comprehension of meaningful messages addressed at the learner, which Krashen (1994, among others) refers to as comprehensible input. This model also posits a sharp distinction between the processes of learning and acquiring a second language. According to the model, the former is a conscious process that encompassed explicit knowledge of rules, whereas the latter encompassed unconscious knowledge of linguistic 
structures and patterns. Furthermore, within Krashen's model it is claimed that it is only acquired L2 knowledge that accounts for learners' fluency in the L2, and that learned rules do not become acquired knowledge, and are only available in tasks for which the L2 user has time to monitor her performance.

Both the natural order of morpheme acquisition studies and the Krashen's comprehensible input model have proved to be limited explanations of L2 learning. This is so because of the limited set of structures for which natural orders have been identified, and because of lack of evidence that comprehension of meaningful messages alone leads to successful L2 learning of linguistic structures (Ellis, 2008). Notwithstanding, the overarching issues of those early studies have been reframed and have evolved as a core problem for second language research. The main issues are whether or not the type of explicit, declarative knowledge of the L2 morphosyntactic rules may be accessed in spontaneous communication, or else is the type of representation that supports accurate usage of morphosyntactic structures in such communication circumstances dependent on implicit linguistic knowledge repositories (Nassaji, 2017).

Implicit language knowledge and learning may be incorrectly understood as involving incidental learning, or learning without attention. Incidental learning as a form of statistical learning has been shown to be a viable explanatory mechanism for L1 acquisition (Winter and Reber, 1994; Erickson and Thiessen, 2015). However, the equivalence between implicit learning and incidental learning is not necessarily established in L2 acquisition studies. According to Schmidt (2010) and Robinson et al. (2014), although L2 learning without overt intention and without availability of any conscious learning effort might occur, there is little evidence that L2 learning takes place without attention to the linguistic input provided by the learning task or the communicative situation. The role of attention in L2 learning is further developed by Ellis' (2006) proposal that theallocation of attentional resources in language processing is a cognitive operation supported by implicit knowledge. Ellis (2006) suggests that the L2 user's language learning history builds up implicit attentional control strategies that will lead her to attend to certain features in the L2.

Ellis' (2006) view of the allocation of attentional resources as implicitly learned attention leads to the hypothetical possibility that attention can be refocused to certain grammatical cues, therefore leaving room to the planning of pedagogical tasks that may support such refocusing, for example exemplarbased and corpus-driven language learning tasks (Boulton, 2009). Unlike Krashen (1994), the perspective put forward by Ellis does not imply a strict division between conscious learning and unconscious acquisition. Declaratively represented linguistic rules could in theory also support the allocation of attentional resources to L2 input processing events, provided conditions for access to those explicit representations are available. Moreover, persistent and continued practice might lead to the automatization of the relevant attentional focus, eventually proceduralizing detection and access to the relevant linguistic cues over the course of L2 communicative events. 
However, a further problem needs to be addressed when it comes to assessment of the validity of presentation of pedagogical grammar rules. Westney (1994, p. 74) defines the notion of rule as "an observed regularity with predictive power". However, as the author points out, language rules should be subdivided into rules of formation and rules of use. Whereas the former express purely distributional properties of morphemes and other linguistic units, the latter tend to entail subtle details of meaning and speaker choice. Westney (1994) claims that when a linguistic regularity is available as pedagogical grammar rule (not all linguistic regularities observable in fluent usage are), its presentation oftentimes accurately describes rules of formation, but it also oftentimes may fail to be appropriately informative of the subtle details and properties subsumed by the corresponding rules of use. The author also suggests that whereas rules of formation can easily be taught and learned through explicit instruction, rules of use may require intensive exposure and use of the L2 for them to become native-like.

Westney (1994) echoes Selinker's (1972) proposal that one of the possible causes for L2 users' interlanguage fossilization, i.e.: failure to restructure flawed L2 representations irrespective of corrective feedback, may stem from transfer of training. According to Selinker (1972), transfer of training is defined as the detrimental effect of instruction on the forms that become available in a bilingual's internalized representation of the L2 linguistic system, that is, her interlanguage system. We understand, following Westney's (1994) argumentation, that the learning of rules of formation without sufficient learning of the rules of use may lead to non-native-like L2 manifestations. Such is the case of the so-called causative-have construction pedagogical rule as described by Vilela's (2009) work.

Vilela (2009) investigated how English native speakers and PortugueseEnglish late bilinguals both comprehended and produced the causative-have construction. This group of bilinguals is interesting because their first language expresses the same meaning and function with an SVO structure coupled with a subject-beneficiary meaning (Ciríaco, 2014). Hence, they have to accommodate the knowledge of this form to that of the second language, the SAuxOV. In her tasks, bilinguals showed higher sensitivity than monolinguals to the use of the SVO with subject-beneficiary meaning. Although that is the correct form in Portuguese, bilinguals seemed to attach to the rule learned by instruction, that the SVO form with this meaning is not possible; whereas monolinguals reported that, even though it is not common, it can occur in their language. The author understood these results as a function of transfer of training: the pedagogical input associated to little linguistic use led to the non-native-like L2 behavior observed.

We now pass to a description of the causative-have construction and the meanings it entails. The pattern is widely taught in schools and textbooks of English as a second language, but not as much attention has been given to it in descriptive and theoretical linguistics. Vilela (2009) reviewed its characterization in textbooks and defined it as having the causative sense and as passive, explaining that just as in the canonic passive, there is a restructuring in the sentence. This view is questioned by Santos (2019) and by the present paper. Instead, according 
to Construction Grammar (Goldberg, 1995; 2006) and following the description of its equivalent in Portuguese (Ciríaco, 2014), it is argued that the clausal pattern is an independent combination of form - $\mathrm{S}$ Aux $\mathrm{O} \mathrm{V}$ - and meaning - provision of services. Nonetheless, both analyses agree that the subject has the semantic role of beneficiary, excluding cases in which the subject has the role of 'maleficiary', such as in We had our roof blown off, or is an agent, as in The teacher had the students write an essay.

An important part of its specification is the auxiliary, expressed by the light verbs have or get. Have seems to be the most prototypical one, as it normally designates the pattern, and it is the most emphasized in L2 teaching. The use of get, however, has shown to be very productive in native language use as well, as the results of this work indicate. Despite that, only few sources, such as the textbook Touchstone (McCarthy et al., 2014) puts HAVE and GET on the same level, calling the structure causative have and get.

Moreover, although it is not elsewhere mentioned, Goldberg (1995) discussed the use of SVO forms such as She painted the house (her example) with the meaning that someone else, not the subject, performed the action of painting. This use is very similar to the one of Portuguese. Goldberg explained that this is possible in given conventionalized scenarios, where there is an intermediate cause. This view is in accordance with Ciríaco's description of the Portuguese transitive construction of agent-beneficiary subject. Both acknowledge the fact that there is an intervening cause in these scenarios, and that the subject has a degree of agency, although it is actually the beneficiary of the action, in Portuguese and in English. However, the use of the SVO is hardly ever taught as possible in English as a Second Language teaching, and in the Brazilian context, it is even more emphasized as an incorrect form.

Hence, the causative-have construction may be very revealing for the investigation of transfer of training in bilinguals interlanguage. As the pedagogical input is so incisive in the teaching of this construction (SAuxOV) and in the rejection of the SVO with a subject beneficiary meaning, even though it seems to be possible (Goldberg, 1995), the distribution of these two patterns in bilinguals' and monolinguals' use may confirm or reject this transfer hypothesis. Additionally, the comparison between comprehension and production data might contribute to understanding the refinements of the effect.

\section{Material and methods}

51 participants were recruited through a chain-referral sampling: 31 Brazilian Portuguese-English bilinguals and 20 English (American) monolinguals. In order to assess their processing of the English causative-have construction, two experiments were conducted over the internet. The first was an interpretation task with the target sentences followed by a 5 point Likert scale, and the second a cloze task, in which the construction was elicited. Before the experiments, bilingual participants performed a speeded version of the Vocabulary Levels 
Test VLT (Nation, 1990), to verify proficiency and thus ability to proceed to the experimental tasks. As VLT informs 5 ranges of vocabulary sizes, in this study, participants were accepted with VLT 3 or more, which is equivalent to knowing the 5000 most frequent lemmas in English. This cut-off point was used for all the words in the experiments.

\subsection{Experiment 1}

The main objective of Experiment 1 was to compare how bilinguals and monolinguals interpreted the construction in the SVO form, as in "Anna cut her hair yesterday." We sought to know if the groups would differ in the semantic role assignment. That is, if they assigned the role of agent or beneficiary for sentences such as the previous. For that reason, participants had to agree with an interpretation sentence, as "She cut her hair herself", on a scale of 1 to 5.

There were 65 experimental items, 18 critical and 47 distractors. The critical items were equally divided into targets (3) and control items (4), adding up to 9 items each.

(3) a. Alice is going to be maid of honor at a wedding tonight.

b. This morning, she did her nails.

c. Alice did her nails herself.

(4) a. Anna gets used to things very easily.

b. This year, she is having her house painted again.

c. Anna painted the house herself.

Every item consisted of three sentences. The first one was meant to contextualize the scenario of the need for a service. Second, there was the target sentence, for the target item this was the SVO form and, for the control, it was the canonical SAuxOV form. The third sentence was the interpretation with which participants were told to agree, on the scale of 1 to 5 that followed. Distractor items had a similar structure but no analysis intent as shown in (5).

(5) a. Linda is very busy this week.

b. This morning, she is making a lot of phone calls.

c. Linda is making a phone call right now.

All the words were controlled for frequency, being among the 5000 most frequent of the English language, according to the Contemporary Corpus of American English (COCA, Davies, 2008-).

Control and target items were meant to be compared, as a means of checking adequate design. Because control items had the canonical form of the construction, they should have very low ratings for a subject agent interpretation, for both groups. Conversely, target items were the ones with the SVO form, which translates literally to Portuguese, and they were meant to inform whether 
bilinguals and monolinguals processed differently the causative-have construction in the comprehension. Participants were told that there was no right or wrong answer, and that they were supposed to choose what they felt was 'natural.'

As in the work of Vilela (2009), we expected that the group of bilinguals would show more sensitivity than monolinguals to the use of the SVO form with a subject-beneficiary interpretation, due to the influence of the pedagogical input. This would be perceived if bilinguals consistently chose smaller numbers in the Likert scale, meaning that they did not agree with the subject agent interpretation (Subject did it -self). This would indicate that the linking rule expressed by the construction, i. e., that a service provided to oneself is associated with the SAuxOV form, which is learned by instruction, is directly transferred to their language use. Such transfer characterizes an interlanguage which is different from the language of monolinguals, for they would not be as sensitive to the use of the SVO associated with the subject-beneficiary interpretation (as perceived by smaller ratings).

Moreover, the verbs of the critical items were further divided into three ranges of frequency among those. Verbs from 1 to 1500 most frequent words were considered of high frequency, the ones from 1501 to 2500 of medium frequency and the ones ranging from 2501 to 5000 of low frequency. This frequency arrangement intended to investigate a possible effect of frequency in the semantic role assignment. We expected that highly frequent verbs would allow the subject beneficiary interpretation.

\subsection{Experiment 2: Cloze Task}

Experiment 2 consisted of a cloze task, in which participants were asked to complete sentences freely. Its main objective was to investigate speakers' production of the construction. This task was carried out in the same online platform as that of Experiment 1, and immediately after it. There were 20 experimental items, 5 targets and 15 distractors. (6) is an example of target and (7) of a distractor.

(6) Marianne is going to the salon this afternoon to

(7) Early today, Barbara went to the grocery shop to

The five target items had an animated subject and a contextual predicate that indicated going to a place where services can and are commonly solicited, such as "the salon" in example (6). Then, they finished with the preposition TO, in order to elicit a verbal form.

We expected that the answer would fall into one of the categories: i) the canonical causative-have: S Aux O V, with HAVE or GET as the auxiliary; ii) the transitive form: S V O, which is the one used in Portuguese or, occasionally, iii) any other response. A first analysis revealed that in some of the instances, the passive construction was also used, hence it was added to the tagging and analysis. 
We expected, following the effect found by Vilela, that both bilinguals would produce more instances of the SVO than of the S Aux O V, while monolinguals would produce more S Aux O V instances. Nonetheless, because of the results of Vilela (2009) and the reporting of the pattern by Goldberg (1995), we also expected monolinguals would produce transitive forms.

\section{Results}

\subsection{Results of Experiment 1}

Our hypothesis for Experiment 1 was that bilinguals would evaluate the subject-beneficiary interpretations as significantly less acceptable than monolinguals would, as Vilela (2009) found. We also expected that control items would be rated less possible in that interpretation, for both groups. As to the frequency, we believed that the highly frequent verbs would favor the subject beneficiary interpretation.

In order to analyze the ordinal data obtained, the ordered numbers in the scale, we built a cumulative mixed model fitted with a Laplace approximation (Christensen, 2018). The model computed ratings in function of the interaction between our conditions: control/target; high/medium/low verb frequencies; and bilingual/monolingual. This model was significant $(\mathrm{p}<0.01)$. It provided us with 66 contrasts, amongst which only 15, the ones where only one condition varied, were taken into consideration in our analysis.

First, all the control items were significantly less acceptable in the subjectbeneficiary interpretation, for both groups, as hypothesized. That indicates that the experiment was adequately designed. Then, we proceeded to the comparisons of verb frequencies, for each group, bilinguals and monolinguals, only in the target items. We wanted to check if highly frequent verbs, such as cut, would favor the subject-beneficiary interpretation more than less frequent verbs of medium and low frequencies, such as redecorate. The results of these comparisons were contrary to our hypothesis, but rather quite interesting. For monolinguals, although we expected the opposite, 1 highly frequent verbs favored the subject-agent interpretation when in SVO sentences, over medium and low frequencies. That indicates that frequency plays a role in the comprehension of the construction, and that the constructions are in complementary distribution when it comes to their function - more frequent verbs will appear in a transitive form (SVO) when the function is agentive, but in a causative-have (SAuxOV and $\mathrm{SVO}$ ) when the function is that of provision of services. In other words, the more frequent verbs are, the more they seem to be associated to the canonical form (SAuxOV) when the subject-beneficiary interpretation is intended, leaving the transitive form (SVO) for subject-agent interpretation.

For bilinguals, there was no effect of frequency whatsoever. This lack of an effect shows that bilinguals and monolinguals are differently affected by frequency, or it may even indicate that for this construction bilinguals are not 
affected by frequency at all. This is probably due to the fact that bilinguals usually learn their L2 mostly through instruction, having little significative real language usage to rely their learning process on. Hence, that could also be seen as an effect of transfer of training, for teaching methods are based solely on outdated and fossilized grammatical exercises which do not incorporate data of real usage, as corpus based materials.

The comparison between bilinguals and monolinguals was the most important aspect in this experiment, because our intention was to find an effect of training in bilinguals' interlanguage. Following Vilela's (2009) findings, we expected that, because of the overruling instruction - that a service provided to a person can only be expressed by the causative-have construction -, bilinguals would consistently reject the use of the transitive form (SVO) associated with subject-beneficiary interpretation. Especially, we hypothesized that they would reject it more than monolinguals, who learned the structure through real usage and not instruction. Again, our results differed from our hypothesis. In the high and low frequencies, there was no significant difference between monolinguals and bilinguals in the interpretation of target sentences. There was a significant effect in the medium range, but what it showed was that bilinguals accepted the subject-beneficiary interpretation more than monolinguals. Thus, our results showed an influence of L1 in L2, not a transfer of training per se. However, the result was different when the task concerned production, as was the case of the second experiment.

\subsection{Results of Experiment 2}

To analyze the data from Experiment 2, we carried out a descriptive statistical analysis. First, we compared the percentage of use and of no use of the construction among groups, this last one including the use of the transitive construction (SVO) as well. Then, for cases in which the construction was used, we accounted for the choice of the auxiliary, have or get.

Table 1 shows bilinguals' and monolinguals' use of the construction, in its canonical form - the SAuxOV -, or in its peripheral form - the transitive, SVO. Bilinguals varied in their use: in items 1, 2 and 3 they preferred the canonical structure, learned by instruction; but on item 4, they primarily chose the SVO, the form of their L1. In items 3 and 5, this group was more evenly divided. They seemed thus to have been influenced by the rule they learned, but not in a categorical way.

Monolinguals, on the other hand, tended to opt for the SAuxOV, as expected. But a tendency is indeed an adequate description for their choice, because they were not categorical either. Monolinguals used the transitive form in three of the five items, although always less than the canonical form. Nonetheless, this use is significative, especially in item five, where their use was very close to the one of bilinguals (35, 3 for these and 37,1 for those). Our results are then, very compelling, for they attest the fact that the SVO form associated with a subject- 
beneficiary meaning, the one elicited by the items, is not non-existent in the English language, as pedagogical grammars reinforce.

Table 1: Distribution of construction use for bilinguals and monolinguals ${ }^{2}$

\begin{tabular}{|l|l|l|l|l|}
\hline & \multicolumn{3}{|l|}{ Bilinguals } & \multicolumn{2}{l|}{ Monolinguals } \\
\hline$\#$ & SAuxOV & SVO & SAuxOV & SVO \\
\hline 1 & 57,1 & 22,8 & 76,4 & 0 \\
\hline 2 & 60 & 25,7 & 82,3 & 11,8 \\
\hline 3 & 34,2 & 20 & 100 & 0 \\
\hline 4 & 31,4 & 62,8 & 88,2 & 11,8 \\
\hline 5 & 45,7 & 37,1 & 64,7 & 35,3 \\
\hline
\end{tabular}

When the canonical construction was used, that is, the SAuxOV form, the two groups behaved differently in their choice of auxiliary verb, as Table 2 demonstrates. Bilinguals clearly preferred the verb have, choosing it in 4 of the 5 items, and the only one that did not have more instances of it, was a tie $(17,1)$. In opposition to that, monolinguals tended to use get in all target items. This result is probably a clearer example of transfer of training. As the pedagogical input presents have as the only possible form, or as the most used one (as in the works of Alexander, 1999; Azar, 2001 and Murphy, 2009), bilinguals were mistakenly led by this rule. Native behavior, however, was the opposite of that, with a preference for get. This result shows the importance of adopting usage-based accounts for pedagogical reasons as well.

Table 2: Distribution of auxiliary verbs of SAuxOV use for bilinguals and monolinguals

\begin{tabular}{|l|l|l|l|l|}
\hline & \multicolumn{2}{|l|}{ Bilinguals } & \multicolumn{2}{l|}{ Monolinguals } \\
\hline$\#$ & GET & HAVE & GET & HAVE \\
\hline 1 & 17,1 & 40 & 58,8 & 17,6 \\
\hline 2 & 25,7 & 34,3 & 52,9 & 29,4 \\
\hline 3 & 17,1 & 17,1 & 58,8 & 41,2 \\
\hline 4 & 20 & 11,4 & 52,9 & 35,3 \\
\hline 5 & 20 & 25,7 & 41,2 & 23,5 \\
\hline
\end{tabular}




\section{Discussion}

The transfer of training that had been reported by Vilela (2009), and that we aimed at investigating in the present paper, was not found in Experiment 1. Vilela found that bilinguals were so attached to the rules that they would be more sensitive to its "transgression" than monolinguals, but we could not replicate this effect. In our experiment, bilinguals behaved similarly to monolinguals, at times accepting the subject-beneficiary interpretation and at others rejecting it. The only statistically significant difference was found in the medium frequency band, in which natives rejected this interpretation, contrary to Vilela's results, and bilinguals accepted the subject-beneficiary interpretation more than monolinguals. Thus, our results showed an influence of L1 in L2, not exactly a transfer of training. We understand that the divergence between our finding and hers is due to the nature of the tasks, where hers resemble pedagogical ones, ours tried to avoid this similarity.

However, we found an effect of instruction in Experiment 2, the one of production. In this task, two results led us to this conclusion. First, bilinguals' use of the construction at high rates and their choice for the verb have, when monolinguals chose get. Grammar books privilege the use of have, even name the construction after it, while get is normally related to an informal use (as in Murphy's, 2009). Second, monolinguals' use of the transitive form with a subjectbeneficiary meaning, which is not mentioned by English grammar books, was employed by our monolingual group. This indicated that the instruction about this pattern, with the meaning of provision of services, is misleading. The canonical causative-have form is taught as the only one possible, but evidence such as this points to the fact that the transitive form can be used with the same meaning, as it is in Portuguese.

Thereby, knowing the rule and applying it causes bilinguals to have a nonnative-like language. If they categorically applied it, they would always use the canonical form (SAuxOV), but that does not happen because the rule seems not to be as internalized. Moreover, they would primarily choose an auxiliary verb, have, that is not the one most natural to native speakers, and in this case that was what happened. In fact, what our experiment shows is that when bilinguals ignored the rule they behaved more similarly to monolinguals.

\section{Conclusion}

In this work, we investigated the effects of transfer of training in the interlanguage of late Brazilian Portuguese-English bilinguals, in relation to their processing of the English causative-have construction. In order to do so, we conducted two experiments, one that focused on the comprehension of the pattern and another which addressed its production. We compared the group of bilinguals to a monolingual one, to attest effects of training, because that is the 
primary manner this bilingual community learns their L2, while monolinguals learn mostly in real usage situations.

The results of both experiments point to the fact that the grammatical rule learned by bilinguals is only 'transferred' when there is time to implement it. That explains the differences between our results and that of Vilela's (2009). Although none of our tasks was timed, they were designed to avoid resorting to episodic memory of learning the rule, while the ones of Vilela resembled pedagogical exercises, which privileged that kind of memory. Hence the discrepancy in the results. In the first experiment, we had no effect of training; on the contrary, bilinguals might have been affected by the SVO distribution in their L1. In the second experiment, however, we saw that bilinguals had high rates of usage of the canonical causative-have. This use, however, was heavily modeled by the instruction through which they learned the language. That was seen in the choice of auxiliary verb, mostly have for bilinguals, but get for monolinguals.

In the task that privileged resorting to the rule, the cloze task, bilinguals showed to be affected by the explicit rule learned in classes. However, in the task that disfavored the resorting of grammatical rules, interpretation of sentences, they showed no strict attachment to the rule and, surprisingly, behaved more similarly to native speakers, as we only found significant differences between the two groups in one of the frequency ranges.

This behavior indicates that bilinguals do not implicitly acquire the rule of causative-have, that is, they do not implement it automatically. Rather, its implementation is seen in more controlled tasks, such as the ones of Vilela's and that of Experiment 2. Thus, explicitly given grammatical rules seem not to be implicitly learned, as they are not automatized to be easily retrieved in real life use, on-the-go.

We are, by no means, claiming that not any grammatical rule is learned in an implicit and automatic manner. We have argued that the causativehave construction does not seem to be internalized by L2 learners. We also acknowledge that our methodology is not exhaustive and neither it is studying only one construction. Future research should widen the variety of constructions, while approaching the matter with a variety of experiments. For instance, offline tasks, such as the ones carried out in this study, should be triangulated with experiments of an online nature, such as most of the ones carried out with an eye-tracking equipment. This practice might enable a fruitful investigation, by allowing the comparison between bilinguals' implicit and explicit representations of language. It can also be the case that other constructions may, and probably do, behave differently. Some may be more prompt to be internalized than others. Additionally, future studies should be attentive to frequency effects in L1 and L2 language use, through the use of corpora and along with the analysis of pedagogical material. 
Notes

1. It is important to note that we expected the opposite but, for limitations intrinsic to the study we conducted, verbs were sorted out by general frequency, not by their frequency in the causative-have construction specifically. An analysis of frequency effects considering verbs which are more or less frequent in this specific construction could show different results, and will be object of study in future research.

2. Values do not add to $100 \%$ because this table only accounts for the use of the construction, with the provision of service meaning, and not for the instances of any other response that did not fall into this category.

\section{References}

Alexander, L. (1990). Longman English grammar practice. London New York: Longman.

Azar, B. \& Hagen, S. (2009). Understanding and using English grammar. White Plains, N.Y: Pearson Education.

Boulton, A. (2009). Testing the limits of data-driven learning: Language proficiency and training. ReCALL, 21(1), 37-54.

Christensen, R. H. B. (2018). Cumulative link models for ordinal regression with the $\mathrm{R}$ package Ordinal. Submitted in J. Stat. Software.

Ciríaco, L. S. (2014). A construção transitiva de sujeito agente-beneficiário no português brasileiro. Caligrama: Revista de Estudos Românicos, 19(2), 83-98.

Davies, Mark. (2008-) The Corpus of Contemporary American English (COCA): 560 million words, 1990-present. Available online at https://corpus.byu.edu/coca/.

Dulay, H. C., \& Burt, M. K. (1973). Should we teach children syntax?. Language learning, 23(2), 245-258.

. (1974). Natural sequences in child second language acquisition 1. Language learning, 24(1), 37-53.

Ellis, N. C. (2006). Selective attention and transfer phenomena in L2 acquisition: Contingency, cue competition, salience, interference, overshadowing, blocking, and perceptual learning. Applied Linguistics, 27(2), 164-194.

(2008). The dynamics of second language emergence: Cycles of language use, language change, and language acquisition. The modern language journal, 92(2), 232-249.

Erickson, L. C., \& Thiessen, E. D. (2015). Statistical learning of language: Theory, validity, and predictions of a statistical learning account of language acquisition. Developmental Review, 37, 66-108.

Goldberg, A. E. (1995). Constructions: A construction grammar approach to argument structure. University of Chicago Press.

. (2006). Constructions at work: The nature of generalization in language. Oxford University Press on Demand.

Krashen, S. (1994). The input hypothesis and its rivals. Implicit and explicit learning of languages, 45-77.

McCarthy, M., McCarten, J. \& Sandiford, H. (2014). Touchstone. New York: Cambridge University Press. 
Murphy, R. \& Murphy, R. (2015). English grammar in use : a self-study reference and practice book for intermediate learners students of English. Cambridge: Cambridge University Press

Nassaji, H. (2017). Grammar acquisition. The Routledge handbook of instructed second language acquisition, 205-223.

Nation, I. S. P. (1990). Teaching and Learning Vocabulary. Teaching Methods. United States: Cengage Learning, Inc. Retrieved September, 9, 2017.

Nemser, W. (1971). Approximative systems of foreign language learners. IRALInternational Review of Applied Linguistics in Language Teaching, 9(2), 115-124.

Robinson, P., Mackey, A., Gass, S. M., \& Schmidt, R. (2012). Attention and awareness in second language acquisition. The Routledge handbook of second language acquisition, 247-267.

Santos, C. F. (2019). Processing of the English causative-have Construction by Monolinguals and Brazilian Portuguese - English Bilinguals. Master's Thesis, Universidade Federal de Minas Gerais, Belo Horizonte, Minas Gerais, Brazil.

Schmidt, R. (2010). Attention, awareness, and individual differences in language learning. In W. M. Chan, S. Chi, K. N. Cin, J. Istanto, M. Nagami, J. W. Sew, T. Suthiwan, \& I. Walker, Proceedings of CLaSIC 2010, Singapore, December 2-4 (pp. 721-737). Singapore: National University of Singapore, Centre for Language Studies.

Segalowitz, N. (2010). Cognitive bases of second language fluency. Routledge.

Selinker, L. (1972). Interlanguage. IRAL-International Review of Applied Linguistics in Language Teaching, 10(1-4), 209-232.

Slabakova, R. (2014). The bottleneck of second language acquisition. Foreign Language Teaching and Research, 46(4), 543-559.

Sorace, A. (2011). Pinning down the concept of "interface" in bilingualism. Linguistic approaches to bilingualism, 1(1), 1-33.

Souza, R. A., \&Oliveira, C. S. F. (2017). Are bilingualism effects on the L1 byproducts of implicit knowledge? Evidence from two experimental tasks. Revista de Estudos da Linguagem, 25(3), 1685-1716.

Ullman, M. T. (2001). The neural basis of lexicon and grammar in first and second language: The declarative/procedural model. Bilingualism: Language and cognition, 4(2), 105-122.

Vilela, A.C. (2009). Transferência Linguística e Transferência de Treinamento na Interlíngua do Falante de Português-L1 / Inglês-L2.(Master's Thesis) . Retrieved from http://www.poslin.letras.ufmg.br/defesas/1239M.pdf.

Westney, P. (1994). Rules and pedagogical grammar. Perspectives on pedagogical grammar, 72-96.

Winter, B., \& Reber, A. S. (1994). Implicit learning and the acquisition of natural languages. Implicit and explicit learning of languages, 115-145.

Recebido em: 10/03/2019

Aceito em: 30/07/2019 